\title{
On Packing and Covering Polyhedra in Infinite Dimensions
}

\author{
Luis Rademacher \\ Department of Computer Science and Engineering \\ Ohio State University \\ Columbus, $\mathrm{OH}$ \\ lrademac at cse dot ohio-state dot edu
}

\author{
Alejandro Toriello* \\ Stewart School of Industrial and Systems Engineering \\ Juan Pablo Vielma \\ Georgia Institute of Technology \\ Atlanta, GA \\ atoriello at isye dot gatech dot edu \\ Sloan School of Management \\ Massachusetts Institute of Technology \\ Boston, MA \\ jvielma at mit dot edu
}

January 13, 2016

\begin{abstract}
We consider the natural generalizations of packing and covering polyhedra in infinite dimensions, and study issues related to duality and integrality of extreme points for these sets. Using appropriate finite truncations we give conditions under which complementary slackness holds for primal/dual pairs of the infinite linear programming problems associated with infinite packing and covering polyhedra. We also give conditions under which the extreme points are integral. We illustrate an application of our results on an infinite-horizon lot-sizing problem.
\end{abstract}

Keywords: covering polyhedron, packing polyhedron, infinite linear program, complementary slackness, integral extreme point

\section{Introduction}

Packing and covering polyhedra have been useful tools in optimization to model a wide variety of decision problems involving resource allocation or demand satisfaction. This has certainly been the case in combinatorial optimization, where many fundamental results involve packing and covering polyhedra, particularly in the study of primal/dual pairs of such polyhedra and the integrality of their extreme points. One salient example is König's Theorem, which establishes the duality relationship between the matching and vertex cover polyhedra of a bipartite graph, two polyhedra known to have integral extreme points.

In the decades since, packing and covering polyhedra have been routinely applied in finitedimensional combinatorial optimization. For example, Fulkerson, who called them anti-blocking and blocking polyhedra respectively [15, 16, 17], used them to derive polarity relations in various combinatorial settings and applied them to several fundamental problems in graph theory and

${ }^{*}$ Corresponding author. 
discrete optimization, e.g. the study of perfect graphs and the derivation or re-derivation of duality results such as the max-flow min-cut theorem.

In this paper we study the generalization of packing and covering polyhedra to problems in infinite dimensions. We first consider strong duality results for the infinite linear programming problems (LP) associated with such polyhedra. Unfortunately, the LPs associated with packing and covering polyhedra often have objective functions that are infinite-valued over some or all of the feasible region. An example where such infinite-valued objective functions naturally appear is infinite-horizon planning problems. A complication that arises from infinite objective values is that some solutions attaining an infinite objective value may be more desirable than others but the objective value does not distinguish this. A common approach to resolve this is to introduce discount factors or running averages to force the objective function to be finite-valued and allow meaningful comparison between solutions. However, these approaches introduce strong biases towards earlier or later periods, respectively, which may be undesirable in some situations. To overcome this issue, we consider a notion of optimality that allows for the comparison of solutions with infinite objective values. This notion extends an idea from combinatorial optimization in infinite graphs and utilizes the complementary slackness condition from strong duality to characterize optimality.

In order to implement this idea of using complementary slackness as a notion of optimality, we introduce a primal/dual pairing between infinite packing and covering linear programs that is easy to construct formally, without using algebraic or topological duality. In general, this is only helpful if the proposed pairing is indeed a primal/dual pair in some standard sense. A contribution of our paper is to show that for the case of packing/covering linear programs, complementary slackness makes sense for our proposed primal/dual pairing. Specifically, one can define optimality by means of complementary slackness of a pair of solutions and show that such a pair of solutions exists under reasonable assumptions (Theorem 11). As an application of these tools, we derive properties for the optimal policies of an infinite horizon lot-sizing problem with an undiscounted and infinite-valued objective function.

Our strong duality results are limited to infinite packing and covering polyhedra defined by linear constraints with only a finite number of non-zero coefficients. Extending this result to more general packing and covering polyhedra will likely require more advanced techniques; one possibility is to apply discrete techniques from infinite graph theory. For this reason we consider the integrality of extreme points in infinite packing and covering polyhedra as a first step towards extending these results.

The remainder of this paper is organized as follows. In Section 2 we introduce notation and definitions, and review some previous work. In Section 3 we show the strong duality result, while in Section 4 we show an application of this result to an infinite horizon lot-sizing problem. Finally, in Section 5 we give the result on integrality of extreme points.

\section{Definitions and Previous Work}

For arbitrary sets $\mathbb{W}$ and $L$, we let $\mathbb{W}^{L}$ denote all functions from $L$ to $\mathbb{W}$. This set can also be thought as tuples of elements in $\mathbb{W}$ indexed by $L$ or as the Cartesian product $\prod_{\ell \in L} \mathbb{W}$. Some of the proof techniques will employ the product topology of $\mathbb{W}^{L}$, where convergence is characterized by coordinate-wise convergence. This convergence is also known as point-wise convergence. If $\mathbb{W}$ is a compact topological space, then $\mathbb{W}^{L}$ is compact when endowed with the product topology by Tychonoff's theorem. We also need the following partial converse to the Krein-Milman theorem:

Theorem 1 (Milman, quoted from [14]). If $K$ is a compact convex subset of a locally convex space and if $A \subseteq K$ is such that $K$ is equal to the closure of the convex hull of $A$, then the extreme points 
of $K$ are contained in the closure of $A$.

We also need an appropriate definition of infinite and possibly uncountable sums. Fortunately, because we will only consider sums of non-negative numbers we can use the following straightforward definition.

Definition 2. Let $I$ be an arbitrary and possibly uncountable index set and let $a \in \mathbb{R}_{+}^{I}$. We let $\sum_{i \in I} a_{i}:=\sup _{S: S \subseteq I,|S|<\infty} \sum_{i \in S} a_{i}$.

We can now introduce packing and covering pairs of LPs through the following definition.

Definition 3 (Packing-Covering Pair). Let $I, J$ be arbitrary and possibly uncountable index sets, $A:=\left(a_{i j}\right)_{i \in I, j \in J} \in \mathbb{R}_{+}^{I \times J}$ be a possibly doubly infinite and non-negative "matrix", and $c:=$ $\left(c_{j}\right)_{j \in J} \in \mathbb{R}_{+}^{J}$ and $b:=\left(b_{i}\right)_{i \in I} \in \mathbb{R}_{+}^{I}$ be non-negative and possibly infinite "vectors". We consider the packing-covering pair of LPs given by

$$
\begin{aligned}
&(P) \quad z^{*}=\inf \sum_{j \in J} c_{j} x_{j} \\
& \text { s.t. } \quad \sum_{j \in J} a_{i j} x_{j} \geq b_{i} \quad \forall i \in I \\
& x_{j} \geq 0 \quad \forall j \in J
\end{aligned}
$$

and

$$
\begin{aligned}
& \text { (D) } w^{*}=\sup \sum_{i \in I} b_{i} y_{i} \\
& \text { s.t. } \quad \sum_{i \in I} a_{i j} y_{i} \leq c_{j} \quad \forall j \in J \\
& y_{i} \geq 0 \quad \forall i \in I .
\end{aligned}
$$

The covering polyhedron defined by $A$ and $b$ is the feasible region of $(P)$ given by $\mathcal{P}_{\uparrow}(A, b):=$ $\left\{x \in \mathbb{R}^{J}:(1 \mathrm{~b}-(1 \mathrm{c})\}\right.$ and the packing polyhedron defined by $A$ and $c$ is the feasible region of $(D)$ given by $\left.\mathcal{P}_{\downarrow}(A, c):=\left\{y \in \mathbb{R}^{I}:(2 \mathrm{~b})-2 \mathrm{c}\right)\right\}$. When $A, b$ and $c$ are clear from the context, we use the notation $\mathcal{P}_{\uparrow}$ and $\mathcal{P}_{\downarrow}$.

If $I$ and $J$ are finite, this pair of problems reduces to a traditional finite packing-covering pair. In addition, special infinite versions of this problem have been studied by several authors. [13] studies the extreme points of the packing polyhedron associated with the stable sets of an infinite perfect graph, and [19] does the same for the packing polyhedron associated with matchings and $b$-matchings of a bipartite infinite graph. In a series of papers, Aharoni and his co-authors study duality for several problems in infinite graphs and hypergraphs, including integer and fractional matching [3, 5, 8, 10, 9], connectivity [4, 7] and flows [6]. Another stream of related work is a series of papers by Romeijn, Smith and their co-authors. These papers study extreme points [12, 18] and duality [22, 23] for problems with more general structure, but only with countably infinite $I$ and $J$; i.e. for the case in which both $\mathcal{P}_{\uparrow}$ and $\mathcal{P}_{\downarrow}$ are contained in $\mathbb{R}^{\mathbb{N}}=\prod_{i=1}^{\infty} \mathbb{R}$. When applied to countably infinite packing-covering pairs the results in 12 imply that the extreme points of the finite projections of $\mathcal{P}_{\downarrow}$ converge in the product topology of $\mathbb{R}^{\mathbb{N}}$ to the closure of the extreme points of $\mathcal{P}_{\downarrow}$ and hence, if the extreme points of the finite projections are all integral, then the extreme points of $\mathcal{P}_{\downarrow}$ are also integral. The results in [22, 23] imply that, under some technical conditions, strong duality holds for $(\mathrm{P}) /(\mathrm{D})$ for countable $I, J$. 
While the previous papers are the most related to our results, infinite LP has been widely studied; we refer the reader to [11] for a longer treatment. In particular, many authors consider strong duality results for primal/dual pairs of infinite LPs in uncountable dimensions using topological or measure-theoretic techniques. An example of such a result and its application in operations research can be found in [1, 2, 20, 21]. However, our formulation is more naturally an extension of existing research for the countable case, because our dual constructions do not rely on linear functionals (and thus are not duals in the classical sense) and because we explicitly allow objectives and constraints to evaluate to infinity.

\section{LP Duality}

The following straightforward lemma shows that weak duality holds for $(\mathrm{P}) /(\mathrm{D})$.

Lemma 4 (Weak Duality). $w^{*} \leq z^{*}$

Proof. Let $\bar{x}$ and $\bar{y}$ be feasible for (P) and (D) and let $I^{+}=\left\{i \in I: \bar{y}_{i}>0\right\}$. Then

$$
\begin{aligned}
\sum_{j \in J} c_{j} \bar{x}_{j} & \geq \sum_{j \in J} \bar{x}_{j} \sum_{i \in I} a_{i j} \bar{y}_{i}=\sum_{j \in J} \bar{x}_{j} \sum_{i \in I^{+}} a_{i j} \bar{y}_{i}=\sum_{j \in J} \sum_{i \in I^{+}} a_{i j} \bar{y}_{i} \bar{x}_{j} \\
& =\sum_{i \in I^{+}} \sum_{j \in J} a_{i j} \bar{y}_{i} \bar{x}_{j}=\sum_{i \in I^{+}} \bar{y}_{i} \sum_{j \in J} a_{i j} \bar{x}_{j} \geq \sum_{i \in I^{+}} b_{i} \bar{y}_{i}=\sum_{i \in I} b_{i} \bar{y}_{i} .
\end{aligned}
$$

The first inequality holds by (2b), non-negativity of $\bar{x}$ and the definition of $\sum_{j \in J}$. The first equality is by the definition of $I^{+}$and the second equality holds by the definition of $\sum_{i \in I^{+}}$. The third equality holds because of non-negativity of $a_{i j}, \bar{x}$ and $\bar{y}$, and by the definitions of $\sum_{i \in I^{+}}$and $\sum_{j \in J}$. The fourth equality holds by the definition of $\sum_{j \in J}$ and the second inequality holds by (1b). The last equality is by the definition of $I^{+}$.

While it is possible to show strong duality under certain settings, e.g. [8, 22, 23, there are many cases in which the objective values of every reasonable feasible solution for (P) and (D) are infinite. A common alternative for these cases is to study the existence of primal/dual solutions that satisfy the following definition of complementary slackness [3, 5, 8, 9, 10].

Definition 5 (Complementary Slackness and Optimality). Feasible solutions $x \in \mathcal{P}_{\uparrow}$ and $y \in \mathcal{P}_{\downarrow}$ satisfy complementary slackness if

$$
\begin{array}{lll}
\forall j \in J & \sum_{i \in I} a_{i j} y_{i}=c_{j} \quad \text { or } \quad x_{j}=0 \\
\forall i \in I \quad & \sum_{j \in J} a_{i j} x_{j}=b_{i} \quad \text { or } \quad y_{i}=0 .
\end{array}
$$

We say the primal/dual pair $(x, y) \in \mathbb{R}^{J} \times \mathbb{R}^{I}$ is $C S$ Optimal if and only if $x \in \mathcal{P}_{\uparrow}, y \in \mathcal{P}_{\downarrow}$ and $(x, y)$ satisfies (3). We say $x \in \mathbb{R}^{J}$ is CS Optimal if and only if there exists $y \in \mathbb{R}^{I}$ such that $(x, y)$ is CS Optimal, and vice versa for $y \in \mathbb{R}^{I}$.

The following straightforward lemma shows that CS Optimal solutions have the smallest possible objective values and their existence implies strong duality.

Lemma 6 (Strong Duality from CS Optimality). If the primal/dual pair $(\bar{x}, \bar{y}) \in \mathbb{R}^{J} \times \mathbb{R}^{I}$ is CS Optimal, then $\sum_{j \in J} c_{j} \bar{x}_{j}=\sum_{i \in I} b_{i} \bar{y}_{i}$. If there exists a CS Optimal solution $\bar{x} \in \mathbb{R}^{J}$, then $w^{*}=z^{*}=\sum_{j \in J} c_{j} \bar{x}_{j}$. 
Proof. Directly from the fact that conditions (3) imply equality for the inequalities in the proof of Lemma 4 .

Lemma 6 shows that a CS optimal solution has the best possible objective value. In particular, if $z^{*}<\infty$, then a CS optimal solution is optimal in the traditional sense. Furthermore, as the following example shows, if $z^{*}=\infty$, CS optimality provides some measure of optimality among solutions with an infinite objective value. However, the example also shows that this measure is not perfect.

Example 7. Consider the packing-covering LP pair given by

$$
\begin{aligned}
& z^{*}=\inf \sum_{j \in \mathbb{N}} x_{j} \\
& w^{*}=\sup \sum_{i \in \mathbb{N}} y_{i, i+1} \\
& \text { s.t. } x_{i}+x_{i+1} \geq 1, \quad \forall i \in \mathbb{N} \\
& x_{j} \geq 0, \quad \forall j \in \mathbb{N} \\
& \text { s.t. } y_{j, j+1}+y_{j+1, j+2} \leq 1, \quad j \in \mathbb{N} \\
& y_{12} \leq 1 \\
& y_{i, i+1} \geq 0, \quad i \in \mathbb{N},
\end{aligned}
$$

which corresponds to a minimum vertex cover and maximum matching in a bipartite graph with one vertex of degree one and all other vertices of degree two, a one-sided infinite path. The two sides of the bipartition contain odd and even vertices respectively, with edges between consecutively numbered nodes. Consider the solutions $\bar{x}^{1}, \bar{x}^{2}, \bar{y}^{1}$ and $\bar{y}^{2}$ given by

$$
\begin{aligned}
\bar{x}_{2 j}^{1} & =1 \text { for } j \in \mathbb{N} \text { and } \bar{x}_{2 j-1}^{1}=0 \text { for } j \in \mathbb{N}, \\
\bar{x}_{1}^{2}=\bar{x}_{2 j}^{2} & =1 \text { for } j \in \mathbb{N} \text { and } \bar{x}_{2 j+1}^{2}=0 \text { for } j \in \mathbb{N}, \\
\bar{y}_{2 i-1,2 i}^{1} & =1 \text { for } i \in \mathbb{N} \text { and } \bar{y}_{2 i, 2 i+1}^{1}=0 \text { for } i \in \mathbb{N}, \\
\bar{y}_{2 i-1,2 i}^{2} & =0 \text { for } i \in \mathbb{N} \text { and } \bar{y}_{2 i, 2 i+1}^{1}=1 \text { for } i \in \mathbb{N} .
\end{aligned}
$$

Even though the objective values of all solutions are infinite, $\bar{x}^{1}$ is intuitively "better" than $\bar{x}^{2}$, since $\bar{x}^{2}$ contains all nodes from $\bar{x}^{1}$. Furthermore, $\bar{x}^{1}$ is CS optimal (either $\bar{y}^{1}$ or $\bar{y}^{2}$ satisfy (3) with it), while $\bar{x}^{2}$ is not, because $x_{1}=1$ implies $y_{12}=1$, which in turn implies $x_{1}+x_{2}=1$.

Conversely, $\bar{y}^{1}$ matches all nodes while $\bar{y}^{2}$ leaves node 1 unmatched, and thus $\bar{y}^{1}$ is intuitively "better". However, both solutions are CS optimal, as both satisfy (3) with $\bar{x}^{1}$. CS optimality can distinguish $\bar{x}^{1}$ being qualitatively better than $\bar{x}^{2}$, but cannot distinguish $\bar{y}^{1}$ being qualitatively better than $\bar{y}^{2}$.

For the countably infinite case, [22, 23, show that the limits of solutions that satisfy complementary slackness for certain finite truncations of (P)/(D) also satisfy complementary slackness for $(\mathrm{P}) /(\mathrm{D})$. A similar technique is used in [8] to show that complementary solutions exist for certain fractional matching/covering pairs in hypergraphs, even in the uncountable setting. This proof directly extends to packing-covering problems so we use it to show that complementary solutions for $(\mathrm{P}) /(\mathrm{D})$ exist under the following conditions.

Assumption 1. The following conditions hold:

i) Row Finiteness: For all $i \in I,\left|\left\{j \in J: a_{i j}>0\right\}\right|<\infty$.

ii) Column Finiteness: For all $j \in J,\left|\left\{i \in I: a_{i j}>0\right\}\right|<\infty$.

The appropriate finite truncations of $(\mathrm{P}) /(\mathrm{D})$ and $(x, y)$ for packing-covering problems are given in the following definitions. 
Definition 8. Let $U \subseteq J$ be such that $|U|<\infty$ and $I(U)=\left\{i \in I: a_{i, j}=0 \quad \forall j \in J \backslash U\right\}$. We define the finite truncation of $P$ associated with $U$ as

$$
\begin{aligned}
&(P(U)) \quad w^{*}=\inf \sum_{j \in U} c_{j} x_{j} \\
& \text { s.t. } \quad \sum_{j \in U} a_{i j} x_{j} \geq b_{i} \quad \forall i \in I(U) \\
& x_{j} \geq 0 \quad \forall j \in U
\end{aligned}
$$

and its feasible region as $\left.\mathcal{P}_{\uparrow}(A, b, U):=\left\{x \in \mathbb{R}^{U}: 4 \mathrm{~b}-4 \mathrm{dc}\right)\right\}$. Similarly, we define the truncation of $D$ associated with $U$ as

$$
\begin{aligned}
&(D(U)) \quad z^{*}=\sup \sum_{i \in I(U)} b_{i} y_{i} \\
& \text { s.t. } \quad \sum_{i \in I(U)} a_{i j} y_{i} \leq c_{i} \quad \forall j \in U \\
& y_{i} \geq 0 \quad \forall i \in I(U)
\end{aligned}
$$

and its feasible region as $\left.\mathcal{P}_{\downarrow}(A, c, U):=\left\{y \in \mathbb{R}^{I(U)}:(5 \mathrm{~b})-5 \mathrm{sc}\right)\right\}$. Again, when $A, b$ and $c$ are clear from context, we use the abbreviated notations $\mathcal{P}_{\uparrow}(U)$ and $\mathcal{P}_{\downarrow}(U)$.

Definition 9. For given vectors $x \in \mathbb{R}^{J}$ and $y \in \mathbb{R}^{I}$ and sets $U \subseteq J$ and $V \subseteq I$ we let $x_{U}:=\left(x_{j}\right)_{j \in U}$ and $y_{V}:=\left(y_{i}\right)_{i \in V}$. We then refer to $x_{U}$ and $y_{V}$ as truncations of $x$ and $y$.

An optimal primal dual pair for $(\mathrm{P}(\mathrm{U})) /(\mathrm{D}(\mathrm{U}))$ satisfies the natural finite version of CS optimality from Definition 5, which is given by the traditional complementary slackness conditions from finite LP. Then, it is natural to hope for the following result regarding finite truncations:

1) Infinite primal/dual pair $(x, y) \in \mathbb{R}^{J} \times \mathbb{R}^{I}$ is CS Optimal for $(\mathrm{P}) /(\mathrm{D})$ if and only if finite truncations $\left(x_{U}, y_{I(U)}\right) \in \mathbb{R}^{U} \times \mathbb{R}^{I(U)}$ are optimal for $(\mathrm{P}(\mathrm{U})) /(\mathrm{D}(\mathrm{U}))$ for all finite $U \subseteq J$.

2) If, for each finite $U \subseteq J$ there exists $\left(\bar{x}^{U}, \bar{y}^{U}\right) \in \mathbb{R}^{U} \times \mathbb{R}^{I(U)}$ that are optimal for $(\mathrm{P}(\mathrm{U})) /(\mathrm{D}(\mathrm{U}))$, then there exists $(x, y) \in \mathbb{R}^{J} \times \mathbb{R}^{I}$ that is CS Optimal for $(\mathrm{P}) /(\mathrm{D})$.

The first condition simply aims to give a series of finite conditions (optimality for $(\mathrm{P}(\mathrm{U})) /(\mathrm{D}(\mathrm{U}))$ ) for fixed infinite solution $(x, y) \in \mathbb{R}^{J} \times \mathbb{R}^{I}$. The second condition goes one step further and aims to show existence of an infinite solution $(x, y) \in \mathbb{R}^{J} \times \mathbb{R}^{I}$ based on the existence of a sequence of finite solutions $\left(\bar{x}^{U}, \bar{y}^{U}\right) \in \mathbb{R}^{U} \times \mathbb{R}^{I(U)}$. This last step is significantly more ambitious as it does not require any coordination between the finite solutions. For instance, if finite $U_{1}, U_{2} \subseteq J$ are such that $U_{1} \cap U_{2} \neq \varnothing$ the finite conditions do not require $\bar{x}_{j}^{U_{1}}=\bar{x}_{j}^{U_{2}}$ for $j \in U_{1} \cap U_{2}$. Hence, it is not clear that the finite solutions can be combined to construct the infinite solution. Fortunately, under some compactness assumptions, we can extend Theorem 3.4 of [8] to show that the finite solutions can indeed be combined together into an infinite solution. However, this combination leads to a series of optimality conditions that are only nearly- or pseudo-finite. We begin by proving this modified version of the first result we hoped to achieve and then show the second result.

Proposition 10 (Pseudo-Finite Optimality). Under Assumption 1 the following conditions are equivalent:

i) $(x, y)$ is CS optimal. 
ii) For every finite $U \subseteq J$ we have

$$
\begin{aligned}
x_{U} & \in \mathcal{P}_{\uparrow}(U) \\
y_{I(U)} & \in \mathcal{P}_{\downarrow}(U) \\
\forall j \in U \quad \sum_{i \in I} a_{i j} y_{i} & =c_{j} \quad \text { or } \quad x_{j}=0 \\
\forall i \in I(U) \sum_{j \in U} a_{i j} x_{j} & =b_{i} \quad \text { or } \quad y_{i}=0 .
\end{aligned}
$$

iii) For every finite $U \subseteq J$ we have

$$
\begin{aligned}
x_{U} & \in \mathcal{P}_{\uparrow}(U) \\
y_{I(U)} & \in \mathcal{P}_{\downarrow}(U) \\
\forall j \in U \quad \sum_{i \in I(U)} a_{i j} y_{i} & =c_{j}^{\prime} \quad \text { or } \quad x_{j}=0 \\
\forall i \in I(U) \sum_{j \in U} a_{i j} x_{j} & =b_{i} \quad \text { or } \quad y_{i}=0 .
\end{aligned}
$$

where $c_{i}^{\prime}=c_{i}-\sum_{i \in I \backslash I(U)} a_{i j} y_{i}$.

Proof. We only prove the equivalence between i) and ii) as the equivalence between ii) and iii) is straightforward.

To see that $x \in \mathcal{P}_{\uparrow}$ is equivalent to $x_{U} \in \mathcal{P}_{\uparrow}(U)$ for all finite $U \subseteq J$ first note that by the definition of $I(U)$ we have $\sum_{j \in U} a_{i j} x_{j}=\sum_{j \in J} a_{i j} x_{j}$ for all $i \in I(U)$. Hence $x$ satisfies the inequality of $\mathcal{P}_{\uparrow}$ indexed by $i \in I(U)$ if and only if $x_{U}$ satisfies the corresponding inequality of $\mathcal{P}_{\uparrow}(U)$. The equivalence then follows by noting that the row finiteness assumption implies that for all $i \in I$ there exist a finite $U \subseteq J$ such that $i \in I(U)$. Similarly, by column finiteness and non-negativity of the $a_{i j}$ we have that $y \in \mathcal{P}_{\downarrow}$ is equivalent to $y_{I(U)} \in \mathcal{P}_{\downarrow}(U)$ for all finite $U$.

Finally, the equivalence of the first complementary slackness condition is direct and the second one follows by again noting that $\sum_{j \in U} a_{i j} x_{j}=\sum_{j \in J} a_{i j} x_{j}$ for all $i \in I(U)$.

The pseudo-finite optimality conditions are very close to complementary slackness conditions for the finite truncations $(P(U)) /(D(U))$. Indeed, if we replace $c^{\prime}$ by $c$ in condition $(7)$ we precisely obtain the optimality conditions of the truncation $(P(U)) /(D(U))$. However, even if this replacement were valid or inconsequential, Proposition 10 does not yield a way to prove existence of an infinte CS optimal primal/dual pair from the existence of finite optimal primal/dual pairs: it only shows that a fixed infinite primal/dual pair satisfies CS optimality if and only if its finite truncations are optimal for a modification of the finite truncations. To show that the finite pairs can indeed be combined into an infinite pair we need the following assumption, guaranteeing certain compactness requirements in the proof.

Assumption 2. We assume $\sup _{i \in I: a_{i j}>0}\left\{b_{i} / a_{i j}\right\}<\infty$ for all $j \in J$ and $\sup _{j \in J: a_{i j}>0}\left\{c_{j} / a_{i j}\right\}<\infty$ for all $i \in I$.

Under this assumption, we can extend Theorem 3.4 of [8] to show that existence of optimal primal/dual pairs for $(P(U)) /(D(U))$ for every finite $U$ do indeed imply the existence of at least one infinite CS optimal pair. Furthermore, certain properties of the finite pairs are guaranteed to be inherited by this infinite pair. To describe such properties we consider sets $F, G \subseteq \mathbb{R}$ representing a 
restriction on the domain of the solutions. For example, $F=G=\mathbb{Z}$ represents the constraint that solutions are integral. Using this we can show that, if all truncations $(P(U)) /(D(U))$ have optimal pairs taking values in $F, G$, then $(P) /(D)$ has a CS Optimal primal/dual pair that also takes values in $F, G$.

Theorem 11. Let $F, G \subseteq \mathbb{R}$ be closed subsets such that $0 \in F, G$. Suppose for any $U \subseteq J$ with $|U|<\infty$ there exist $x^{U} \in F^{U} \cap \mathcal{P}_{\uparrow}(U)$ and $y^{U} \in G^{I(U)} \cap \mathcal{P}_{\downarrow}(U)$ such that

$$
\begin{array}{lll}
\forall j \in U \quad \sum_{i \in I(U)} a_{i j} y_{i}^{U}=c_{j} & \text { or } & x_{j}^{U}=0 \\
\forall i \in I(U) \quad \sum_{j \in U} a_{i j} x_{j}^{U}=b_{i} & \text { or } & y_{i}^{U}=0,
\end{array}
$$

where the superscript indicates the solutions' dependence on the set $U$. Then, under Assumptions 1 and 2, there exists a primal dual pair $(x, y) \in F^{J} \cap \mathcal{P}_{\uparrow} \times G^{I} \cap \mathcal{P}_{\downarrow}$ of CS optimal solutions for $(P) /(D)$.

Proof. We follow and extend the proof of a similar result in $[8$ for fractional matchings in infinite graphs and hypergraphs. Let $g_{j}:=\sup _{i \in I: a_{i j}>0}\left\{b_{i} / a_{i j}\right\}, h_{i}:=\sup _{j \in J: a_{i j}>0}\left\{c_{j} / a_{i j}\right\}$, and let $X:=$ $\prod_{j \in J}\left[0, g_{j}\right], Y:=\prod_{i \in I}\left[0, h_{i}\right]$. By Assumption 2 and Tychonoff's Theorem, $X \times Y$ is compact. Furthermore, $\mathcal{P}_{\downarrow} \subseteq Y$ and every $x^{U} \in F^{U} \cap \mathcal{P}_{\uparrow}(U)$ satisfying (8) is contained in $\prod_{j \in U}\left[0, g_{j}\right]$ (because such $x^{U}$ is optimal for $P(U)$ ).

Now, for any finite $U \subseteq J$, let

$$
C(U)=\left\{\begin{array}{rlrl}
x_{U} & \in \mathcal{P}_{\uparrow}(U), & y & \in \mathcal{P}_{\downarrow}, \\
(x, y) \in X \times Y: & \in F^{J} \times G^{I}, & (x, y) \text { satisfies (3) }
\end{array}\right\}
$$

By the assumptions, $C(U)$ is closed. To see that it is non-empty take $x \in F^{U} \cap \mathcal{P}_{\uparrow}(U)$ and $y \in G^{I(U)} \cap \mathcal{P}_{\downarrow}(U)$ satisfying (8) and extend them to $X \times Y$ by appending zeros. Family $C(U)$ has the finite intersection property because for any finite subfamily $\left(U_{i}\right)_{i}$ we have $C\left(\bigcup_{i} U_{i}\right) \subseteq \bigcap_{i} C\left(U_{i}\right)$. Then, by compactness we have that $\bigcap_{U \subseteq I:|U|<\infty} C(U) \neq \varnothing$. To obtain the desired result it just remains to prove that if $x \in F^{J}$ is such that $x_{U} \in \mathcal{P}_{\uparrow}(U)$ for all $U \subseteq I$ with $|U|<\infty$, then $x \in \mathcal{P}_{\uparrow}$. This follows by row finiteness (condition $(i)$ of Assumption 1), by noting that each constraint of $\mathcal{P}_{\uparrow}$ is a constraint of $\mathcal{P}_{\uparrow}(U)$ for a sufficiently large but finite $U \subseteq I$.

\section{Application to Lot Sizing}

We next discuss our results' application to an infinite-horizon version of the single-item lot-sizing problem [22]. For this we assume there is an infinite sequence of demands $b_{t}>0$ for $t \in \mathbb{N}$ that must be met either with product produced in the same period or with product held over in inventory from previous periods; backlogging is not allowed. The assumption that demand is positive is made for ease of exposition and can be relaxed. There are time-dependent unit production and holding costs, $c_{t}>0$ and $h_{t}>0$ respectively. Instead of the traditional inventory balance formulation, we employ assignment variables $x_{s t}$ that indicate how much of period $t$ 's demand is produced in period $s \leq t$; these variables have a composite unit cost of $c_{s t}=c_{s}+\sum_{\tau=s}^{t-1} h_{\tau}$. This naturally leads to the 
formulation

$$
\begin{aligned}
& \inf \sum_{s=1}^{\infty} \sum_{t=s}^{\infty} c_{s t} x_{s t} \\
& \text { s.t. } \sum_{s=1}^{t} x_{s t} \geq b_{t}, \quad t \in \mathbb{N} \\
& x_{s t} \geq 0 \quad s=1, \ldots t, \quad t \in \mathbb{N}
\end{aligned}
$$

The model clearly satisfies Assumptions 1 and 2 , and the dual is

$$
\begin{aligned}
& \sup \sum_{t=1}^{\infty} b_{t} y_{t} \\
& \text { s.t. } y_{t} \leq c_{s t}, \quad s=1, \ldots, t, \quad t \in \mathbb{N} \\
& y_{t} \geq 0, \quad t \in \mathbb{N}
\end{aligned}
$$

Applying Theorem 11 and our definition of CS optimality, we can derive the following conclusions. Let $x^{*}$ and $y^{*}$ be CS optimal; since $b_{t}>0$, some $x_{s t}^{*}$ must be positive, and therefore

$$
\begin{array}{rlrl}
y_{t}^{*} & =\min _{s \leq t} c_{s t} & t \in \mathbb{N} & \\
x_{s t}^{*}>0 & \Rightarrow y_{t}^{*}=c_{s t} & s=1, \ldots, t \quad t \in \mathbb{N}
\end{array}
$$

That is, production to meet demand in period $t$ should only occur in the period(s) $s \leq t$ that affords the cheapest overall unit cost. Since $c_{t}>0$, 10a in turn implies $y_{t}^{*}>0$ and hence

$$
\sum_{s=1}^{t} x_{s t}^{*}=b_{t} \quad t \in \mathbb{N}
$$

i.e. each period's demand is met exactly. Finally, a simple calculation shows

$$
c_{s t}=\min _{\sigma \leq t} c_{\sigma t} \Rightarrow c_{s \tau}=\min _{\sigma \leq \tau} c_{\sigma \tau}, \quad \tau=s, \ldots, t,
$$

and therefore if it is optimal to produce in period $s$ for period $t$, it is also optimal to produce in $s$ for all intervening periods. This reasoning gives the following result, an extension of [22, Theorem 5.1] for the case when $(9)$ does not necessarily have a finite optimal value.

Theorem 12. For problem (9), we can choose a CS optimal production plan $x^{*}$ with a production epoch or regeneration interval structure:

$$
x_{s t}^{*}>0 \Rightarrow x_{s \tau}^{*}=b_{\tau}, x_{\sigma \tau}^{*}=0, \quad \sigma \in\{1, \ldots, \tau\} \backslash\{s\}, \quad \tau=s, \ldots, t .
$$

\section{Extreme Point Structure}

The topological techniques used in Section 3 are strongly dependent on the finite row/column conditions of Assumption 1. While duality results have been proven in the context of infinite graphs [3, 5, 8, 10] without these assumptions, the proofs require more elaborate techniques that are usually connected to the discrete structure of these problems. This suggests that extending duality results in the absence of Assumption 1 might be possible for infinite LPs with discrete 
structures or solutions. In this section we study the existence of such discrete structures in infinite LPs as a first step towards this extension. Theorem 11 already shows that CS optimal solutions can inherit discrete structures of the optimal solutions of the truncations. For instance, if all truncations have integral optimal solutions $(F=G=\mathbb{Z})$, then there exists an integral CS optimal solution. We show that a similar inheritance holds even in the absence of Assumption 1. More precisely, we give a generalization of a result in [13] to show that the extreme points of $\mathcal{P}_{\uparrow}$ and $\mathcal{P}_{\downarrow}$ inherit the properties of the following finite truncations.

Definition 13. For $U \subseteq J$ and $V \subseteq I$, let

i) $\widetilde{\mathcal{P}_{\uparrow}}(U):=\left\{x \in \mathbb{R}^{U}: \sum_{j \in U} a_{i j} x_{j} \geq b_{i} \quad \forall i \in I, \quad x_{j} \geq 0 \quad \forall j \in U\right\}$,

ii) $\widetilde{\mathcal{P}_{\downarrow}}(V):=\left\{y \in \mathbb{R}^{V}: \sum_{i \in V} a_{i j} y_{i} \leq c_{j} \quad \forall j \in J, \quad y_{i} \geq 0 \quad \forall i \in V\right\}$.

\subsection{Packing Polyhedra}

Theorem 14. Let $F \subseteq \mathbb{R}$ be closed and such that $0 \in F$. If for any finite $V \subseteq I$ the extreme points of $\widetilde{\mathcal{P}_{\downarrow}}(V)$ are in $F^{V}$, then under Assumption 2 the extreme points of $\mathcal{P}_{\downarrow}$ are in $F^{I}$.

Proof. We follow and extend the proof of a similar result in [13] for stable set polyhedra of infinite perfect graphs (See Definition 15). Equip $\mathbb{R}^{I}$ with the product topology; this ensures it is a locally convex, Hausdorff space. Assume $\left(a_{i j}\right)_{j \in J}$ contains at least one non-zero for each $i$, implying the boundedness (and compactness) of $\mathcal{P}_{\downarrow}$. The general case is a simple extension.

The conclusion follows from Theorem 1 with $K=\mathcal{P}_{\downarrow}$ and $A=F^{I} \cap \mathcal{P}_{\downarrow}$, a closed set. It is enough to show that the closure of the convex hull of $A$ contains $\mathcal{P}_{\downarrow}$, and we show this now. Let $\hat{y} \in \mathcal{P}_{\downarrow}$, and consider any open set containing $\hat{y}$. Let $V \subseteq I$ be the finite index set of coordinates for which this open set's component is not equal to $\mathbb{R}$. Define $\bar{y} \in \mathcal{P}_{\downarrow}$ as $\bar{y}_{j}=\hat{y}_{j}$ for $i \in V$ and 0 otherwise. Then $\bar{y}_{V} \in \mathcal{P}_{\downarrow}(V)$, and hence it is a convex combination of points in $F^{V} \cap \mathcal{P}_{\downarrow}(V)$. By extending with zeros in all other coordinates, we can take this combination also in $\mathbb{R}^{I}$; thus $\hat{y}$ is in the closure of the convex hull of points in $A$.

Theorem 14 has several direct corollaries. To describe them we will need the following definitions concerning (infinite) graphs.

Definition 15. A (infinite) graph is a pair $G=(V, E)$ where $V$ an arbitrary and possibly uncountable set and $E \subseteq\{\{u, v\}: u, v \in V, u \neq v\}$. For $U \subseteq V$ we let $G(U)=\{U, E(U)\}$ be the graph induced by $U$, where $E(U):=\{\{u, v\} \in E: u, v \in U\}$. For $v \in V$ we let $E(v):=\{e \in E: v \in e\}$.

- We say a graph $G$ is perfect if every one of its finite induced graphs is perfect.

- We say an graph $G=(V, E)$ is bipartite if there exist $U_{1}, U_{2} \subseteq V$ such that $U_{1} \cap U_{2}=\varnothing$, $V=U_{1} \cup U_{2}$ and $E \subseteq\left\{\left\{u_{1}, u_{2}\right\}: u_{1} \in U_{1}, u_{2} \in U_{2}\right\}$.

- The stable set polyhedron of a graph $G$ is given by

$$
\left\{y \in \mathbb{R}^{V}: y_{v}+y_{u} \leq 1 \quad \forall\{u, v\} \in E, \quad y_{v} \geq 0 \quad \forall v \in V\right\}
$$

- The fractional matching polyhedron of $G$ is given by

$$
\left\{y \in \mathbb{R}^{E}: \sum_{e \in E(V)} y_{e} \leq 1 \quad \forall v \in V, \quad y_{e} \geq 0 \quad \forall e \in E\right\} .
$$


- For $b \in \mathbb{Z}_{+}^{V}$, the fractional $b$-matching polyhedron of $G$ is given by

$$
\left\{y \in \mathbb{R}^{E}: \sum_{e \in E(V)} y_{e} \leq b_{v} \quad \forall v \in V, \quad y_{e} \geq 0 \quad \forall e \in E\right\} .
$$

- The fractional vertex cover polyhedron of $G$ is given by

$$
\left\{x \in \mathbb{R}^{V}: x_{u}+x_{v} \geq 1 \quad \forall\{u, v\} \in E, \quad x_{v} \geq 0 \quad \forall v \in V\right\} .
$$

With these definitions we can obtain some well known results from [13, 19].

Corollary 16 ([13]). The stable set polyhedron of a perfect graph has integer extreme points. The extreme points of the fractional matching polyhedron of a graph are half-integral.

Furthermore, we can extend some well known results concerning finite graphs to the infinite setting.

Corollary 17 ([19]). The b-matching polyhedron of a bipartite graph has integer extreme points.

\subsection{Covering Polyhedra}

Similarly to Theorem 14, we may consider solutions with values in certain closed sets containing 0 . However, for simplicity we restrict our attention to integral solutions.

Theorem 18. If for any finite $U \subseteq J$ the extreme points of $\widetilde{\mathcal{P}_{\uparrow}}(U)$ are integral, then, under Assumption 2, the extreme points of $\mathcal{P}_{\uparrow}$ are integral.

Proof. Let $d_{j}:=\left\lceil\sup _{i \in I: a_{i j}>0}\left\{b_{i} / a_{i j}\right\}\right\rceil+1$. The proof is similar to the proof of Theorem 14. Equip $\mathbb{R}^{J}$ with the product topology, and consider the set $\mathcal{P}_{\uparrow} \cap\left\{x \in \mathbb{R}^{J}: x_{j} \leq d_{j}, \forall j \in J\right\}$. Using the same argument from the previous proof with $\bar{x}_{j}=d_{j}, \forall j \in J \backslash J^{\prime}$, it follows that this set has integer extreme points, since the upper bound constraints cannot add fractional extreme points. Moreover, any $x \in \mathcal{P}_{\uparrow}$ having some $j \in J$ with $x_{j}>d_{j}$ cannot be extreme, so the extreme points of this truncated set contain the extreme points of $\mathcal{P}_{\uparrow}$.

Similarly to Theorem 14, Theorem 18 yields as a corollary the following result.

Corollary 19. The extreme points of the fractional vertex cover polyhedron of a graph are halfintegral.

\section{Acknowledgements}

The authors thank the associate editor and reviewer for their positive, constructive feedback, which greatly improved the manuscript. L. Rademacher's work was supported by the National Science Foundation under grants 1350870 and 1422830. 


\section{References}

[1] D. Adelman and D. Klabjan, Duality and Existence of Optimal Policies in Generalized Joint Replenishment, Mathematics of Operations Research 30 (2005), 28-50.

[2] — Computing Near-Optimal Policies in Generalized Joint Replenishment, INFORMS Journal on Computing 24 (2011), 148-164.

[3] R. Aharoni, König's Duality Theorem for Infinite Bipartite Graphs, Journal of the London Mathematical Society 29 (1984), 1-12.

[4] R. Aharoni, Menger's theorem for countable graphs, J. Comb. Theory, Ser. B 43 (1987), no. 3, 303-313.

[5] R. Aharoni, Infinite matching theory, Discrete Mathematics 95 (1991), 5-22.

[6] R. Aharoni, E. Berger, A. Georgakopoulos, A. Perlstein, and P. Sprüssel, The max-flow min-cut theorem for countable networks, J. Comb. Theory, Ser. B 101 (2011), no. 1, 1-17.

[7] R. Aharoni and R. Diestel, Menger's theorem for a countable source set, Combinatorics, Probability \& Computing 3 (1994), 145-156.

[8] R. Aharoni and R. Holzman, Optimal fractional matchings and covers in infinite hypergraphs: Existence and duality, Graphs and Combinatorics 8 (1992), 11-22.

[9] R. Aharoni and V. Korman, Strong LP duality in weighted infinite bipartite graphs, Discrete Mathematics 131 (1994), no. 1-3, 1-7.

[10] R. Aharoni and R. Ziv, LP duality in infinite hypergraphs, Journal of Combinatorial Theory, Series B (1990), 82-92.

[11] E.J. Anderson and P. Nash, Linear Programming in Infinite-Dimensional Spaces, John Wiley \& Sons, Inc., Chichester, 1987.

[12] W.P. Cross, H.E. Romeijn, and R.L. Smith, Approximating extreme points of infinite dimensional convex sets, Mathematics of Operations Research 23 (1998), 433-442.

[13] R.C. Dalang, On Infinite Perfect Graphs and Randomized Stopping Points on the Plane, Probability Theory and Related Fields 78 (1988), 357-378.

[14] V.P. Fonf, J. Lindenstrauss, and R.R. Phelps, Infinite dimensional convexity, Handbook of the Geometry of Banach Spaces (W.B. Johnson and J. Lindenstrauss, eds.), vol. 1, Elsevier Science B.V., 2001, pp. 599-670.

[15] D.R. Fulkerson, Blocking Polyhedra, Graph Theory and its Applications (B. Harris, ed.), Academic Press, New York, 1970, pp. 93-112.

[16] _ Blocking and Anti-Blocking Pairs of Polyhedra, Mathematical Programming 1 (1971), 168-194.

[17] _ Anti-Blocking Polyhedra, Journal of Combinatorial Theory 12 (1972), 50-71.

[18] A. Ghate and R. L. Smith, Characterizing extreme points as basic feasible solutions in infinite linear programs, Operations Research Letters 37 (2009), 7-10. 
[19] R. Grzkaślewicz, On Extreme Infinite Doubly Stochastic Matrices, Illinois Journal of Mathematics 31 (1987), 529-543.

[20] D. Klabjan and D. Adelman, Existence of Optimal Policies for Semi-Markov Decision Processes Using Duality for Infinite Linear Programming, SIAM Journal on Control and Optimization 44 (2006), 2104-2122.

[21] _ A Convergent Infinite Dimensional Linear Programming Algorithm for Deterministic Semi-Markov Decision Processes on Borel Spaces, Mathematics of Operations Research 32 (2007), 528-550.

[22] H.E. Romeijn and R.L. Smith, Shadow prices in infinite-dimensional linear programming, Mathematics of Operations Research 23 (1998), 239-256.

[23] H.E. Romeijn, R.L. Smith, and J.C. Bean, Duality in infinite dimensional linear programming, Mathematical Programming 53 (1992), 79-97. 